Virginia Commonwealth University

vCU Scholars Compass

2010

\title{
Nonclassical crystallization of amorphous iron nanoparticles by radio frequency methods
}

Kyler J. Carroll

Virginia Commonwealth University

J.A. Pitts

Virginia Commonwealth University

Kai Zhang

Norfolk State University

A. K. Pradhan

Norfolk State University

E. E. Carpenter

Virginia Commonwealth University, ecarpenter2@vcu.edu

Follow this and additional works at: http://scholarscompass.vcu.edu/chem_pubs

Part of the Chemistry Commons

Carroll, K. J., Pitts, J. A., \& Zhang, K., et al. Nonclassical crystallization of amorphous iron nanoparticles by radio frequency methods. Journal of Applied Physics, 107, 09A302 (2010). Copyright (C) 2010 American Institute of Physics.

\section{Downloaded from}

http://scholarscompass.vcu.edu/chem_pubs/32

This Article is brought to you for free and open access by the Dept. of Chemistry at VCU Scholars Compass. It has been accepted for inclusion in Chemistry Publications by an authorized administrator of VCU Scholars Compass. For more information, please contact libcompass@vcu.edu. 


\title{
Nonclassical crystallization of amorphous iron nanoparticles by radio frequency methods
}

\author{
K. J. Carroll, ${ }^{1}$ J. A. Pitts, ${ }^{1}$ Kai Zhang, ${ }^{2}$ A. K. Pradhan, ${ }^{2}$ and E. E. Carpenter ${ }^{1, a)}$ \\ ${ }^{1}$ Department of Chemistry, Virginia Commonwealth University, Richmond, Virginia 23284, USA \\ ${ }^{2}$ Center for Materials Research, Norfolk State University, Norfolk, Virginia 23504, USA
}

(Presented 19 January 2010; received 31 October 2009; accepted 20 November 2009; published online 15 April 2010)

\begin{abstract}
Amorphous iron nanoparticles were synthesized using an aqueous reduction in iron(II) sulfate with sodium borohydride and sodium citrate. Various radio frequency (rf) exposure times were investigated in order to determine trends in nonclassical crystallization. RF times from 15 to $300 \mathrm{~s}$ revealed an increase in crystallite size from 5 to $60 \mathrm{~nm}$, as determined by powder x-ray diffraction. Also, solvent optimization revealed that ethanol produced the largest trends for increasing crystallite size without total oxidation of the samples. Magnetic characterization by room temperature vibrating sample magnetometry and high resolution transmission microscopy was performed to verify magnetic properties and particle morphology. () 2010 American Institute of Physics. [doi:10.1063/1.3334169]
\end{abstract}

Single-crystal monodisperse ferromagnetic nanoparticles are an interesting area of research for their use in electronic and biomedical devices. Several papers have highlighted classical methods, such as the self-assembly of nanostructures, for producing crystalline monodisperse ferromagnetic materials. ${ }^{1-9}$ Amorphous metal nanostructures offer a nonclassical approach to creating crystalline monodisperse nanoparticles by not relying on the nucleation and growth process but by inducing crystallization into pre-synthesized particles. ${ }^{10}$ This work shows a novel way to induce crystallation into amorphous metals by radio frequency (RF) techniques. RF methods allow for tunable more controlled heating to orient atoms from an amorphous state to a destabilized crystalline state within a nanostructure. ${ }^{11,12}$ Clusters of about $100 \mathrm{~nm}$ amorphous iron nanoparticles were synthesized by aqueous reduction using sodium borohydride and sodium citrate. $^{13}$

The synthesis of Fe nanoparticles was carried out under ambient conditions. A borohydride to metal ratio and a metal to citrate ratio were kept constant at $2: 1$ and 10:1, respectively. In a typical experiment $4.60 \mathrm{mM}$ iron(II) sulfate heptahydrate and $0.460 \mathrm{mM}$ trisodium citrate dihydrate were mixed vigorously for 10 min using a magnetic stir-bar in $2 \mathrm{~L}$ of DI $-\mathrm{H}_{2} \mathrm{O}$ to ensure total dissolution. Sodium borohydride $(8.80 \mathrm{mM})$ was added to the mixture and allowed to react for $10 \mathrm{~min}$. The particles were magnetically washed several times with ethanol. After washing, the particles were placed in a vacuum oven to dry overnight prior to rf exposure.

$\mathrm{RF}$ exposure was done on an Ameritherm HOTSHOT 2 $\mathrm{kW}$ rf generator. Approximately $0.1 \mathrm{~g}$ of the as-prepared powder was placed in a glass test tube. The solvent was then added to the test tube $(5 \mathrm{ml})$ and mixed to disperse the particles. Initial and final temperatures of the solvent were recorded. After exposure the particles were collected by magnetic separation, and then washed with ethanol (three times).

${ }^{a)}$ Electronic mail: ecarpenter2@vcu.edu.
The sample was then dried in a vacuum oven prior to characterization.

The dried powders were characterized by several techniques for the determination of phase, morphology, and temperature dependent magnetic properties. A typical high resolution transmission electron microscopy (TEM) image of the as-prepared amorphous nanoparticles is shown in Fig. 1. From the size distribution histogram (inset), an average particle size of $104 \pm 1.9 \mathrm{~nm}$ could be estimated. A relatively small distribution of particle size was seen. About 50 particles were considered to obtain the size distribution histogram. Also, the image (inset) shows a lighter shell around the particles which is an amorphous $\mathrm{Fe}^{2+}$ determined by $\mathrm{x}$-ray photoelectron spectroscopy (XPS).

High resolution photoelectron spectra were recorded using an ESCALAB 250 spectrometer. $\mathrm{An}^{+}{ }^{+}$ion flood gun was used for charge compensation and an ion etch gun $(3 \mathrm{kV}$, $3 \mathrm{~mA}$ ) was used for depth profiling. XPS was conducted on the as-prepared particles to determine surface composition. Figure 2(B) shows the atomic percentage of $\mathrm{Fe}^{2+}$ and $\mathrm{Fe}^{0}$ as

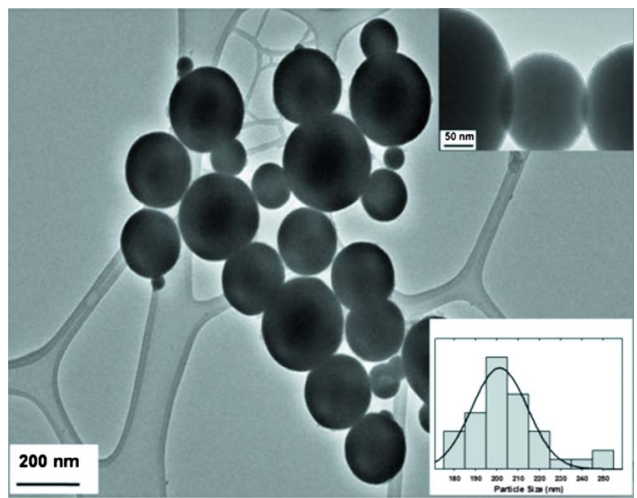

FIG. 1. (Color online) TEM image of the as-prepared amorphous nanoparticles, (top right) image shows a thin layer of $\mathrm{Fe}^{2+}$ surrounding the nanoparticles, (bottom right) histogram of particle size for the as-prepared particles. 


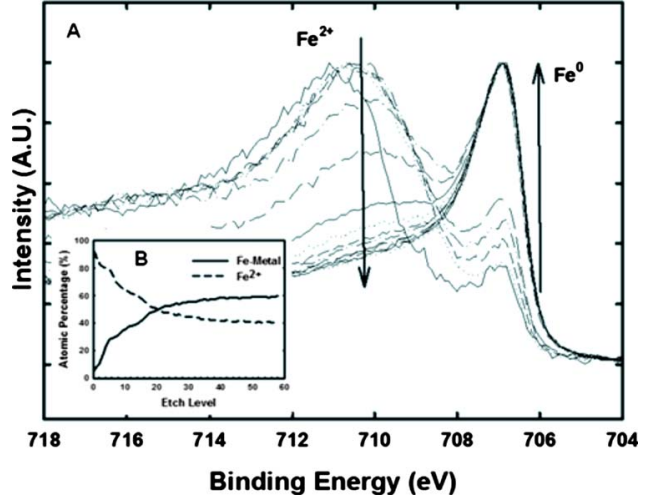

FIG. 2. (Color online) (a) Depth profile of the Fe $2 p$ XPS region spectra. (b) Atomic percentage vs etch level which shows an decrease in $\mathrm{Fe}^{2+}$ and an increase in Fe-metal as the etch level increases. This suggests that at the surface a layer of oxide is present on the particles.

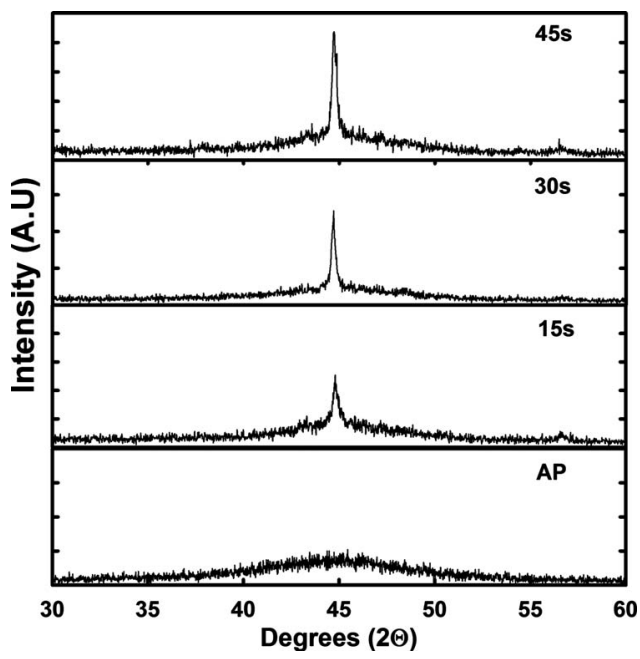

FIG. 3. XRD patterns at various rf exposure times. From the graph it can be seen that as the exposure time increases the $45^{\circ}$ Bragg diffraction peak increases.

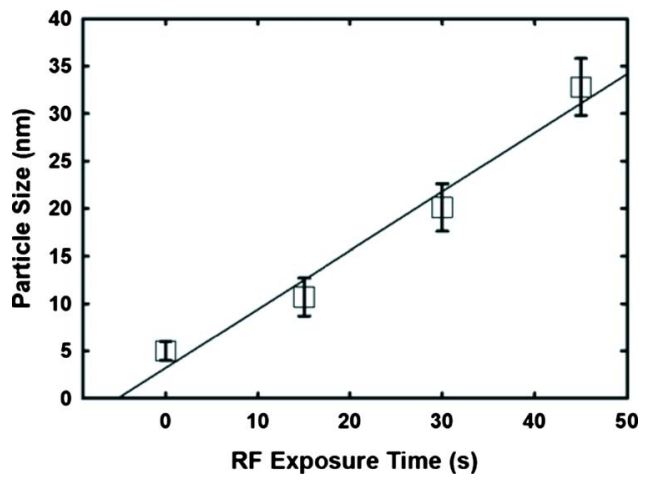

FIG. 4. (Color online) Graph of particle size vs rf exposure time.

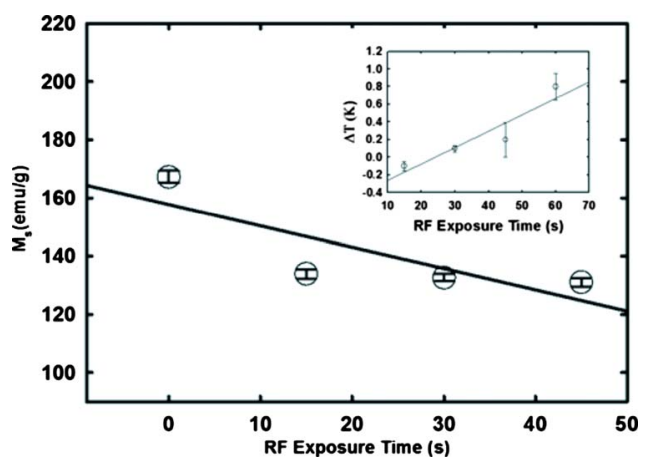

FIG. 5. (Color online) Graph of saturation magnetization vs rf exposure time with statistical significance, (top right) graph of $\Delta \mathrm{T}$ vs rf exposure time.

a function of etch levels. From Fe $2 p$ region scan, it can be seen that the surface of the particle consists mainly of $\mathrm{Fe}^{2+}$ and the concentration of $\mathrm{Fe}^{2+}$ decreases as the surface is sputtered, exposing a higher concentration of $\mathrm{Fe}^{0}$. The $\mathrm{C} 1 \mathrm{~s}$ spectra reveal three peaks, which is consistent with a surface with adsorbed citrate. The peaks are consistent with a $\mathrm{C}-\mathrm{C}$, $\mathrm{C}-\mathrm{O}$, and $\mathrm{C}=\mathrm{O}$ bonds. While the $\mathrm{C} 1 \mathrm{~s}$ spectra speculates citrate on the surface, the exact type of interaction is uncertain because of the large scan area used in the analysis. Also, the surface roughness introduced by having spherical particles does not produce even surface etching.

Figure 3 represents a series of powder x-ray diffraction (XRD) patterns obtained on the dried powders at different $\mathrm{rf}$ exposure times. In Fig. 3, an increase in crystallinity is seen from no rf exposure to an rf exposure of $45 \mathrm{sec}$. The Scherrer equation was used to determine the crystallite size changes versus the rf exposure time (Fig. 4). For example, the crystallite size increases from 5 to $30 \mathrm{~nm}$ for particles with 0 to $45 \mathrm{~s}$ of $\mathrm{rf}$ exposure, respectively.

Figure 5 represents saturation magnetization versus rf exposure time. From this graph it can be seen that a decrease in saturation magnetization is seen as the rf exposure time is increased. The change in temperature of the solvent versus rf exposure time was also investigated (inset). From this graph a linear correlation is seen which can be explained by the particles heating in the rf field causing the solvent to increase in temperature. The error bars represent the uncertainty in the temperature measurements.

An aqueous synthesis of $\mathrm{Fe}(\mathrm{II})$ sulfate using sodium borohydride and sodium citrate produced spherical monodisperse amorphous nanoparticles which served as the starting material for the study of nonclassical crystallization. These results show promise in the area of $\mathrm{rf}$ techniques for the production well defined single-crystal nanoparticles for electronic and biomedical devices. Ethanol as a solvent helped to dissipate heat to and from the particles for crystallization which prevented agglomeration and oxidation. In this nonclassical method, the rf technique provided enough energy to the Fe atoms to cause them to reorient to a more crystalline state.

\section{ACKNOWLEDGMENTS}

This research was supported in part by the VCU Nanomaterials Core Characterization Facility, School of Engineer- 
ing Foundation and a grant from NSF under Grant No. CHE0820945 MRI (XPS).

${ }^{1}$ A. P. Alivisatos, Science 271, 933 (1996).

${ }^{2}$ P. Buffat and J. P. Borel, Phys. Rev. A 13, 2287 (1976).

${ }^{3}$ U. Cho, Y. Lee, S. Kumar, C. Lee, and B. Koo Sci. China, Ser. E: Technol. Sci. 52, 19 (2009).

${ }^{4}$ P. Y. Keng, I. Shim, B. D. Korth, J. F. Douglas, and J. Pyun, ACS Nano 1, 279 (2007).

${ }^{5}$ V. Shulika, A. Potapov, and N. Noskova, Phys. Met. Metallogr. 104, 228 (2007).

${ }^{6}$ D. Sudfeld, K. Wojczykowski, W. Hachmann, S. Heitmann, K. Rott, T.
Hempel, S. Kammerer, P. Jutzi, A. Hutten, and G. Reiss, IEEE Trans. Magn. 38, 2601 (2002).

${ }^{7}$ S. Sun and C. B. Murray, J. Appl. Phys. 85, 4325 (1999).

${ }^{8}$ S. Sun, C. B. Murray, D. Weller, L. Folks, and A. Moser, Science 287 , 1989 (2000).

${ }^{9}$ J. R. Thomas, J. Appl. Phys. 37, 2914 (1966).

${ }^{10}$ M. W. Grinstaff, M. B. Salamon, and K. S. Suslick, Phys. Rev. B 48, 269 (1993).

${ }^{11}$ M. Kopcewicz, Struct. Chem. 2, (105)313 (1991).

${ }^{12}$ P. Kwapulinski, Z. Stoklosa, J. Rasek, G. Badura, G. Haneczok, L. Pajak, and L. Lelatko, J. Magn. Magn. Mater. 320, e778 (2008).

${ }^{13}$ L. Zhang and A. Manthiram, J. Appl. Phys. 80, 4534 (1996). 